\title{
La violence en Centre éducatif fermé : enjeux et traitement institutionnel
}

\author{
Léo Farcy-Callon
}

Le sociographe, vol. 56, no. 4, 2016

Le thème de la violence est si vaste qu'une tentative de définition ou de conceptualisation paraîtrait démesurée. Les études portant sur ce thème peuvent autant s'intéresser aux conflits armés qu'aux violences politiques, aux émeutes urbaines, ou aux violences conjugales. Les violences peuvent prendre des formes très variées et se distinguer dans leurs pratiques, leurs natures, leurs finalités (Crettiez, 2008). Nous nous intéresserons ici à un cas particulier, les violences ayant lieu au sein des organisations du secteur sanitaire et social, se caractérisant par leur spécificité propre au secteur.

L'existence de violences au sein de tous types d'institutions est aujourd'hui admise. La grande majorité des organisations scolaires, sociales, sanitaires, pénales connaissent leur lot de domination, d'agressivité ou de montée en tension. Il apparait cependant que certains secteurs et certaines structures rencontrent ces phénomènes de façon quotidienne. Le cas des institutions de la protection de l'enfance et de la justice des mineurs est caractéristique d'établissements confrontés à ces violences de manière récurrente et répétée (Bailleau, Gourmelon et Milburn, 2012 ; Barreyre, 2008 ; Potin, 2014).

S'il existe une surreprésentation de violences physiques dans les établissements du secteur sanitaire et social, nous verrons qu'elle ne se limite pas à ces formes. En effet, nous considérons que celles-ci peuvent être physiques, morales ou symboliques. Physiques, car la forme la plus visible de violence est corporelle. Morales, de par la prévalence des menaces, des insultes et de toute autre forme d'agression verbale. Et enfin symboliques, au travers des tensions subjacentes et des pressions pouvant être vécues par les acteurs. Nous retenons, dans ce travail, une définition large de la violence : une action directe ou indirecte, consciente ou inconsciente limitant ou blessant les personnes ou les biens (Nieburg, 1969).

Nous souhaitons dans ce travail questionner la complexité de ces violences en prenant l'exemple des Centres éducatifs fermé ${ }^{1}$, établissements accueillant des mineurs condamnés ou placés par la justice pénale. Cet écrit s'organise en trois parties. Tout d'abord, il s'agira de décrire une journée d'immersion où les violences ont été particulièrement présentes et visibles. Ensuite, nous présenterons les tenants et les aboutissants de ces violences. Comment se caractérisent-elles ? Pourquoi sont-elles si présentes ? Comment émergentelles ? Enfin, nous appréhenderons les réponses proposées par l'institution à ces violences.

\footnotetext{
${ }^{1}$ Les Centres éducatifs fermés (CEF) sont créés par la loi 2002-1138 du 9 septembre 2002. Celle-ci crée ces nouveaux établissements éducatifs dans le but d'accueillir des mineurs âgés entre 14 et 17 ans, multirécidivistes ou multiréitérants. Sont placés en CEF les mineurs ayant été soumis à une décision de contrôle judiciaire ou ceux condamnés à une peine assortie d'un sursis avec mise à l'épreuve. Depuis la loi du 10 mars 2004, les mineurs en liberté conditionnelle ou en aménagement de peine peuvent également y être placés. Pour cette dernière, la décision de placement intervient au cours de la détention.
} 
Comment les professionnels les contiennent, les apaisent et les régulent ? Quels outils peuvent-ils mobiliser pour les canaliser?

Nous nous appuierons, pour cela, sur les premiers résultats d'une thèse de sociologie portant sur les pratiques professionnelles dans les établissements de la justice pénale des mineurs. Notre enquête procède d'une méthode ethnographique et s'appuie sur des supports empiriques variés : collectes de données quantitatives (consultation de dossiers de mineurs, de projets associatifs, de projets d'établissements, de projets de service, etc.), observation in situ (avec la participation à la vie des établissements et à des temps de travail en équipe), entretiens semi-directifs (avec des mineurs et des professionnels), et «focus group » (ou méthode d'analyse en groupe) (Van Camenhoudt, Chaumont et Franssen, 2005).

\section{Vivre la violence en Centre éducatif fermé}

Les Centres éducatifs fermés sont des lieux où les violences sont omniprésentes, que ce soit par l'imposition d'une contrainte à ses publics et ses professionnels ou par les scènes quotidiennes qui s'y déroulent (Mucchielli, 2005). En effet, nous pouvons tout d'abord attester de la présence excessive de violences visibles : bagarres, insultes, etc. Il se joue cependant d'autres formes de violences plus subtiles et moins visibles. Ces mécanismes symboliques sont moins évidents à percevoir dans des temps d'observation in situ, mais ressortent davantage dans le discours des acteurs, jeunes comme intervenants.

Il est, à première vue, difficile d'appréhender la nature de ces violences. Pour ce faire, nous proposons de partir d'une étude de cas, d'une scène vécue lors d'une immersion dans un Centre éducatif fermé associatif. Cela nous permettra, d'une part, de mieux visualiser les logiques d'agressivité et de pouvoir, et d'autre part, de comprendre les stratégies développées par les institutions et les intervenants sociaux pour apaiser les ardeurs.

\section{Extrait de carnet de terrain}

«Ce matin, mon arrivée au CEF est plutôt particulière. À peine entré dans le jardin, une éducatrice me salue, et me lance d'une voix stressée et rapide et courant vers sa voiture $\mathrm{Ha}$ vous êtes là. Hé bien vous avez bien choisi votre journée! Sans vraiment comprendre, je franchis le portail et m'en vais saluer les éducateurs et les jeunes présents sous le kiosque, dans le jardin, en train de fumer. Bonjour, bonjour, quelques échanges courtois, etc. Je n'ose pas vraiment demander aux éducateurs ce qu'il se passe en présence des jeunes, mais je sens bien une tension sous-jacente. Un des jeunes est dans son coin, ne parle à personne. Pourtant la dernière fois que je l'ai vu il était plutôt bavard. Les éducateurs parlent entre eux de choses et d'autres. Quelques minutes plus tard, alors que je me rends au bureau des éducateurs pour poser mon sac, l'institutrice m'explique que l'ambiance est particulièrement tendue. Elle m'apprend qu'une éducatrice s'est fait agresser par un jeune la veille. S'est fait agresser peut vouloir dire beaucoup de choses et laisse la place à toute mon imagination. Je n'ai pas plus de détail, car l'institutrice part en classe. Je décide de ne pas m'arrêter sur cet événement et ne pas trop poser de questions directes sur le sujet. Je descends donc dans le jardin et rejoins le groupe.

Digression : La journée a suivi son cours, et j'ai appris ce qu'il s'était vraiment passé au compte-goutte, chacun me donne des détails de son point de vue. J'ai ensuite, à la fin de la journée, en rentrant chez moi, pu réunir l'ensemble des témoignages des jeunes et des professionnels et pu reconstituer plus ou moins l'événement. L'éducatrice serait rentrée 
dans la chambre de Maxime, un des jeunes, et celui-ci l'aurait empêchée de sortir. Il aurait fermé la porte, commencé à l'insulter et à crier, et cela tout en la brusquant physiquement. Le mineur en question est placé pour une affaire de mœurs. Si j'ai bien compris, l'éducatrice a paniqué du fait du passé de ce mineur. Il n'y a pas eu d'agression sexuelle d'aucune sorte, mais le fait d'enfermer l'éducatrice est déjà un acte grave. Elle a de plus reçu quelques coups au passage. Ils ont appelé ça une séquestration. Le gamin a tout de suite été envoyé en garde à vue. D'après les professionnels, il est foutu, il va partir directement en prison. Quand on agresse un éducateur, on ne peut pas rester. Les professionnels sont chargés d'une mission de service public. Donc les juges sont intransigeants.

Reprise : Vers $9 \mathrm{~h}$, quelques minutes après mon arrivée, les jeunes partent en activité. Cette fois-ci, je n'y participe pas. Je profite que l'infirmière n'a pas de rendez-vous pour faire un entretien avec elle. Je rejoins les jeunes et les autres professionnels à midi pour le repas. Il y a toujours un peu d'eau dans le gaz. Un des jeunes est en train de harceler un éducateur de questions pour savoir où est Maxime, celui ayant agressé l'éducateur. Il veut savoir s'il est en prison. Au fond, je pense qu'il sait, c'est une affirmation qu'il attend : alors il est où ? Il ne va pas revenir ? Il part direct en prison ? Les éducateurs répondent à côté. On ne sait pas, pour l'instant il est en garde à vue, il va passer devant le juge. Ils tentent de contourner le sujet en faisant quelques blagues et en essayant de rigoler avec les jeunes. C'est à ce moment-là que la tension est montée en quelques secondes seulement. Celui qui posait les questions ne s'arrête pas. Un autre jeune se lève pour se resservir, et lui sort un ça va ferme ta "gueule" maintenant d'un air tout naturel. Quoi, t'as dit quoi ? Le premier se lève, retourne la table, les assiettes et les verres tombent, il met un coup de poing au second. Ils s'insultent et se crachent en pleine figure la nourriture qu'ils avaient encore dans la bouche. Un éducateur et le cuisinier réagissent au quart de tour : les deux jeunes sont tout de suite immobilisés (ils leur bloquent les deux bras derrière le dos comme le ferait un agent de police). Lâche-moi "connard", je vais le "niquer". Les insultes fusent, les jeunes se débattent, les professionnels prennent deux trois coups au passage. Puis au bout d'un moment, ça se calme un peu. Mais les esprits sont encore chauds. Ils continuent à s'envoyer quelques insultes. L'éducateur sportif et le cuisinier prennent chacun un jeune à part. J'entends l'éducateur sportif parler en arabe. Ça calme tout de suite le jeune. On ramasse les tables et les chaises. Je remarque alors que certains des autres jeunes avaient eu le réflexe de se mettre tout de suite sur le côté, et d'attendre que ça se calme. Il y en a même un qui est parti s'assoir dans le salon. Il semble avoir l'habitude.

Il me dira d'ailleurs plus tard dans la journée : t'as vu, moi je me suis tout de suite barré. Ça ne m'intéresse pas ces trucs. Je ne m'occupe plus de ça, après ça nous fout dans la merde parce qu'on croit qu'on fait partie de l'embrouille. Cette précision du jeune, évoquée lors d'un entretien effectué avec lui en fin de journée, m'a particulièrement intéressé et m'a poussé à le questionner plus amplement sur son vécu au CEF. Tu rentres ici, t'as fait une connerie, une seule connerie. T'es calme, t'es un gars calme, t'as fait une connerie, l'erreur est humaine. $\mathrm{Tu}$ arrives ici, tu vois que les gens ils ont fait ces conneries. Ici, tu prends un coup, ici tout le monde prend un coup. N'importe quelle personne qui vient ici, il prend un coup, il prend une frappe. Il devient "con", il commence à devenir fou. Mais fou t'as compris, pas comme quand je rigole avec toi, c'est pour rigoler t'as compris. Mais il prend un coup. Pourquoi ? Parce que t'es violent, t'insultes, je ne sais pas... Dehors, jamais je n'insulte dehors. Tu peux demander à n'importe qui. On est sous pression à mort. Tu vois par exemple tout à l'heure, "ta gueule", péter un plomb, tu vois ? Dehors tu me dirais "ta gueule", mais va y tranquille, OK je ferme ma "gueule". Ici, tu dis "ta gueule", d'entrée ça veut se taper. Cette mise sous pression permanente ne fait qu'accentuer les tensions qui peuvent exister. Ce même jeune 
m'expliquera également pourquoi cette pression ressentie l'amène à être dans de la confrontation permanente. On est ici ils nous guettent. On est derrière ils nous guettent, on est là-bas ils nous guettent, on est aux toilettes ils nous guettent. Wesh au bout d'un moment, tu te douches, Ouais il y a qui ? Qui se douche? Hé ça va! Ça va! Tu veux quoi ? Tu vois ou pas ? Au bout d'un moment, c'est comme ça, les éducateurs tu ne les respectes plus. T'es dans ta chambre, t'es calé, t'es calé au calme. Ils viennent, toc-toc-toc une fois. Bon vas -y tu te dis, ils vérifient si tu es bien dans ta chambre si tu n'es pas parti en fugue. Deux fois, trois fois, quatre fois, cinq fois, wesh au bout d'un moment tu veux que je me mette à poil tu rentres dans ma chambre tu veux me voir à poil ou quoi?

À la fin de la bagarre, tout le monde reprend sa place et ceux dont les assiettes ont volé vont se resservir. Les deux jeunes se serrent la main à la demande de l'éducateur sportif. L'ambiance s'apaise. La suite des événements est alors très étrange : un long silence, au moins 3 minutes. Personne ne dit un mot. Une éducatrice me lance alors ça va ? Le silence perdure. Deux trois rires se font entendre. Les intervenants échangent des regards et se marrent. Le cuisinier lance : putain quelle ambiance ici! Hé ho! Ca va vous êtes là ? Les jeunes commencent à reparler un peu. La même question revient sur le tas : alors il est en prison ou pas ? L'éducateur rigole : mais je n'en sais rien, lâche moi avec ça. Tout le monde retrouve plus ou moins ses esprits. Le repas reprend son cours normal. Un des jeunes ayant participé à la bagarre dit en rigolant au cuisinier : tu m'as bien bloqué quand même! Bien joué ! Je t'aurais. Tu vas voir la prochaine fois qu'on se tape ! Le cuisinier répond : Ouais t'inquiètes, tu vas voir après manger on va se taper. Et tu m'as mis un bon coup de coude dans le nez, mon salaud. »

\section{Violences physiques et violences symboliques}

Cet extrait de carnet de terrain nous laisse percevoir l'intensité de la violence dans ces établissements. La journée en question commence très calmement. La matinée a été extrêmement apaisante pour les professionnels et il suffit d'un rien pour que la violence et les tensions atteignent leur paroxysme. Si les jeunes n'en sont arrivés aux mains qu'au moment du repas, la symbolique de cette violence était, quant à elle, présente depuis le début la journée de par l'agression de la veille. Nous pouvons ici parler de violence symbolique, car le groupe de jeunes placés a pressenti l'exclusion de Maxime comme une forme de domination (Bourdieu et Passeron, 1970), comme un pouvoir que l'institution porte sur eux à leur insu, sans qu'ils n'aient aucune marge de manœuvre. Les questions répétées d'un des mineurs sur la situation de Maxime sont caractéristiques de son identification à ce dernier.

À ce titre, nous pouvons voir cette violence individuelle comme une forme de réponse des jeunes à une violence qu'ils subissent de manière plus générale, car les formes de la délinquance juvénile se rapprochent d'une déviance socialement marquée. Les actes de ces jeunes sont, en partie, les conséquences de leur exclusion et de leur stigmatisation (Mucchielli, 2001, p.76). Leurs parcours de vie sont marqués par des séries de ruptures, avec leur famille, avec l'école, avec les institutions de la justice des mineurs. La rencontre avec la justice est alors l'aboutissement d'un engrenage de prises en charge institutionnelles dû à leur instabilité sociale. Tout ce vécu menant à un placement en CEF s'apparente à une forme de violence. Les intervenants sociaux des CEF sont alors confrontés à des jeunes vivant les placements comme une violence symbolique (Bourquin, 2005).

Un premier moyen de comprendre cette violence est de s'intéresser au vécu de ces jeunes et à la manière dont ils vivent leur histoire institutionnelle. Un second élément nous paraît 
également central pour comprendre les comportements des jeunes : celui du quotidien de l'enfermement. La violence semble être un moyen de briser la routine de l'enfermement, élément mis en lumière par les théories de l'expérience carcérale (Rostaing, 2006).

Les journées se ressemblent dans ces lieux que sont les Centres éducatifs fermés. Du réveil au coucher, en passant par les activités et les repas ${ }^{2}$, le temps est organisé, il est réglé. Les heures prennent alors une allure bien particulière. Régulé par les horaires de travail des professionnels, ce temps amène à un sentiment de routine que seul un moment de crise peut briser. Certains jeunes ont pu me dire au détour de conversations qu'ils tournaient en rond. Ils tentent alors de briser cet ennui du quotidien par n'importe quel moyen : jouer au chat et à la souris avec les éducateurs, voler une cigarette ou un objet qui traine, parfois crier et chercher le conflit. Des fois on se tape parce qu'on s'ennuie a pu me confier un jeune à ce sujet.

La violence semble exacerbée par la violence institutionnelle décrite plus haut et la tension liée à l'enfermement, concrétisée par la surveillance permanente rapportée par un des jeunes ci-dessus, vécue comme un harcèlement, un étouffement et un déficit d'intimité, voire une dépossession de son identité. Tout cet élément nous laisse penser que les Centres éducatifs fermés peuvent être considérés comme des institutions totalitaires (total institution) au sens de Goffman (Goffman, 1968). Sur ce point, un article de Catherine Lenzi et Philip Milburn nous éclaire sur l'emprise mentale que l'institution peut exercer sur les individus, transformant par là même leur identité (Lenzi et Milburn, 2015).

S'ajoute à cela la nature des relations entre jeunes. Les Centres éducatifs fermés accueillent 12 jeunes qui vivent 24 heures sur 24 et 7 jours sur 7 en collectivité. Ils possèdent des chambres individuelles, mais il leur est souvent interdit d'y retourner durant la journée. Comme dans tous les espaces de vie collective, se développent des enjeux de pouvoir et d'appartenance (Goffman, 1968). Il existe souvent plusieurs groupes au sein des centres. Certains sont plus influents que d'autres et ont davantage de pouvoir. Plusieurs jeunes ont pu me confier qu'ils se sentaient souvent sous pression et qu'ils devaient tenir pour ne pas se faire marcher dessus. Cela signifie qu'ils ont un rôle à jouer. Il y a une hiérarchie qui se crée. Celle-ci repose tout d'abord sur la nature des délits et des crimes de chacun. Les plus grands ont fait des braquages, viennent ensuite les dealers et les agresseurs, puis tout en bas de l'échelle les "pointeurs », les auteurs de viols ou d'agressions sexuelles. Il se joue alors des phénomènes subtils où les jeunes racontent leurs exploits de délinquant, méprisent certains comportements et se mettent en scène pour paraître et pour conserver leur position hiérarchique (Caisne [le], 2008). À ce titre, la violence peut également être démonstrative. Il faut se faire voir et se faire respecter devant les autres. Il n'y a rien de mieux qu'une baston pour se faire sa place, m'ont confié plusieurs jeunes à la suite de la bagarre. Ces différents enjeux génèrent des débordements de violence.

«Quand un acteur joue un rôle, il demande implicitement à ses partenaires de prendre au sérieux l'impression qu'il produit. Il leur demande de croire que le personnage qu'ils voient possède réellement les attributs qu'il donne l'apparence de posséder ; que l'activité qu'il exerce aura effectivement les conséquences qu'elle est implicitement censée entrainer, et

\footnotetext{
${ }^{2}$ Les temps de repas sont souvent en effectif réduit du côté des professionnels. Les éducateurs sont au nombre de deux, et il ne reste plus personne sur la structure. Ainsi, ce moment est particulièrement difficile pour deux professionnels face à douze jeunes.
} 
que, d'une façon générale, les choses sont bien ce qu'elles ont l'apparence d'être ». (Goffman, 1973, p.25)

Pour revenir au cas pratique développé plus haut, nous remarquons que les professionnels étaient bel et bien conscients que cette ambiance du matin n'était que le calme avant la tempête. Pour preuve, la première éducatrice rencontrée en entrant dans le CEF prévient d'une situation tendue bien avant que les esprits s'échauffent. Les intervenants sociaux ont conscience de l'existence de ce volcan endormi. Ils ont également conscience de l'existence d'une violence symbolique forte liée au vécu de ces jeunes, aux différentes épreuves (Dubet, 1994) qu'ils ont connues et à l'expérience (Martuccelli, 1999 et 2006) que représente un séjour en Centre éducatif fermé. L'institution et les intervenants sont donc lucides quant à l'action qu'ils peuvent avoir et mettent en place un ensemble de pratiques visant à amenuiser cette action contenante.

\section{Quelle réponse de la part de l'institution?}

\section{Des réponses immédiates et situationnelles}

Nous souhaitons à présent appréhender quelles réponses institutionnelles et professionnelles sont données à ces violences. Tout d'abord, d'un point de vue formel, les CEF ont développé un ensemble de protocoles visant à minimiser l'action contenante qu'ils peuvent avoir sur leur public et, par ce biais, à limiter les comportements des jeunes. Il y a par exemple des sorties très régulières du centre qui leur donnent l'occasion de quitter les murs institutionnels. Les retours en familles sont également fréquents et permettent aux jeunes d'avoir une relation avec le monde de l'extérieur. Cependant, ces réponses de l'institution ne permettent pas d'apaiser les violences dans l'immédiateté de la situation. Face à une altercation entre deux jeunes, il convient, dans un premier temps, de réagir pour calmer la situation et rétablir un ordre social. En effet, face à cette tension générale, l'apaisement paraît quasiment impossible. Les jeunes sont sous pression, ils souhaitent poursuivre cette bagarre, en découdre, et ne sont pas près de laisser les éducateurs les calmer. Pourtant, nous remarquons qu'après les quelques minutes de silence qui ont suivi la scène de violence, l'ambiance se détend.

Tout d'abord, la question de la contention physique est significative. En cas d'excès de violence d'un jeune, les intervenants sociaux peuvent être amenés à faire ce qui est communément appelé de la contention. Très mobilisé dans les institutions de santé mentale, cela se caractérise par l'utilisation de la force physique du professionnel sur le jeune, en le mettant au sol ou en lui faisant une clef de bras. Cela permet de l'immobiliser et de stopper sa crise de violence. Cette pratique fait particulièrement polémique du fait qu'une violence physique est exercée sur le jeune. L'institution est donc très prudente vis-à-vis de ces pratiques et demande à ce qu'elles soient utilisées le moins possible. La contention est à la marge. Ça reste un évènement qui doit rester exceptionnel nous disait un chef de service. En principe, un intervenant ayant pratiqué la contention doit se justifier par écrit et même parfois échanger par téléphone avec le magistrat. Les intervenants usent souvent de la métaphore du parapluie pour décrire ces règles. Ils supposent que l'interdiction de la contention est davantage une protection de la part de l'institution et de l'établissement qu'une véritable idéologie éducative de prohibition de la violence.

Dans cette situation, le rôle du cuisinier nous a paru particulièrement intéressant. Ce dernier prend une place centrale dans la gestion du conflit, puisqu'il se permet de stopper physiquement le jeune pendant qu'il se bat. Il intervient spontanément pour stopper les 
tensions entre les jeunes, alors que son rôle au sein de l'institution n'est ni de maintenir l'ordre ni de travailler avec les jeunes sur leur situation. Nous pouvons faire des rapprochements avec la théorie d'Andrew Abbott sur les jurisdictions (Abott, 1988), parfois traduites en français comme les «territoires » du professionnel (Champy, 2009). Ils sont définis comme les tâches ou fonctions revendiquées par un professionnel dans la division du travail. L'intervention du cuisinier met en lumière la fragilité des territoires de l'intervention socio-éducative et la part informelle de la pratique. En effet, il va agir sur un territoire qui n'est pas le sien, puisque sa fonction se limite initialement à l'intendance alimentaire. Sur ce point, plusieurs travaux de recherche confirment qu'il se joue parfois une distribution informelle des rôles où les intervenants ne se limitent pas à leur fonction prescrite (Chantraine et al, 2008, Frauenfelder, Nada et Bugnon, 2013). Par ailleurs, nous pouvons interpréter l'intervention du cuisinier comme une action de solidarité envers les membres de son équipe. Son but est avant tout de préserver l'institution à laquelle il appartient, et de préserver un ordre social au sein de l'établissement.

Ce qui est particulièrement complexe dans cette pratique de la contention, c'est qu'elle est là en premier lieu pour protéger le jeune, pour l'empêcher de faire un acte qui aurait une conséquence trop grave pouvant notamment entrainer des poursuites pénales. De moins, c'est l'explication que plusieurs intervenants donnent à l'utilisation de la contention. Mais cela peut également être interprété comme un maintien de l'ordre social. Quoi qu'il en soit, tout le paradoxe réside dans cette mise en danger des intervenants pour protéger le jeune. D'autant plus que ce dernier ne demande pas d'aide et qu'au contraire, il n'en souhaite pas.

Dans un autre registre, les professionnels utilisent, dans notre situation, des tactiques qui résident dans l'utilisation de l'humour ou de la camaraderie. Ces stratégies sont développées par Pierre Vidal-Naquet dans un article où il nous présente les différentes « ruses » que les professionnels mettent en place pour détourner l'attention de l'usager (Vidal-Naquet, 2015). Par ce biais, les professionnels entrainent les jeunes dans un climat paisible, détendent l'atmosphère et permettent de passer outre les événements. Nous pouvons percevoir ces ruses dans les blagues que font certains intervenants ou dans l'utilisation de l'arabe par l'un des éducateurs. Comme nous le dit Vidal-Naquet, «le placement de la communication interpersonnelle sur le terrain de la jovialité et de la gaieté participe de ces techniques de dérivation susceptibles de réduire les éventuelles tensions et de renforcer la coopération entre aidants et aidés (ibid., p.7) ». L'utilisation de la plaisanterie est également pointée dans l'ouvrage d'Alice Jaspart concernant les Centres éducatifs fermés belges (Jaspart, 2016). Le rire et la camaraderie deviennent ici des outils de travail, avec cependant le risque d'entrainer une banalisation de la violence devenue acceptable de la sorte.

Nous pouvons ici appréhender l'imprévisibilité du travail (Champy, 2009). Les professionnels s'adaptent à la situation complexe des mineurs et tentent de résoudre un problème dans son immédiateté, quitte à ce que la solution soit instable et partielle, quitte à ce que les rôles prescrits de chacun ne soient pas respectés. Dans le cas de l'action éducative, cela peut être lu comme un évitement des désordres. Quitte à trouver des arrangements précaires, les intervenants sociaux tentent coûte que coûte de garantir l'accompagnement éducatif (Rostaing, 2014). Ils tentent de répondre à une situation qu'ils jugent urgente pour garantir une certaine stabilité sociale au sein de l'établissement. 


\section{Des réponses collectives et informelles}

Il convient à présent de saisir ce qui s'est déroulé après la journée décrite plus haut. À l'issue de ces événements, des délibérations ont eu lieu sur la réponse collective à donner aux jeunes ayant transgressé et s'étant comportés violemment. Pour le cas de Maxime, les forces de l'ordre sont intervenues. Une note est envoyée au juge et le jeune répondra de ses actes devant la justice. Pour la bagarre, la décision est prise qu'aucun écrit ne soit fait, que ce soit à propos des mineurs et de leur comportement ou concernant la contention faite par l'éducateur. L'incident sera géré au sein de l'équipe éducative. Hormis une discussion de recadrage et une demande d'excuse, les jeunes ne seront pas sanctionnés. Les intervenants ne souhaitent pas prévenir les autorités judiciaires, notamment pour ne pas aggraver leur situation pénale.

Le fait de porter plainte peut faire une affaire qui le fera basculer pour quelques années aussi. Qu'est-ce qu'on cherche ? Sinon je peux lancer toute la machine, je fais un dépôt de plainte tout ça. Après... on lance la machine judiciaire une fois de plus sur ce gamin (éducateur).

Les résultats d'une recherche menée collectivement au sein de plusieurs Centres éducatifs fermés ${ }^{3}$ ont mis en lumière le fait que les professionnels mobilisent des espaces de travail intermédiaires ou clandestins (Lenzi, Milburn et al, 2015). Ils créent des espaces d'ouverture, des espaces de liberté pour garantir une fonction éducative. Cette « pédagogie de la responsabilisation » (Milburn, 2009) nous montre que les intervenants sociaux sont sans cesse amenés à faire appel aux valeurs des jeunes sans forcément se reposer sur la toute-puissance de l'institution. La subjectivation peut donc, parfois, substituer l'assujettissement au sein du travail socio-éducatif (Roux, 2012). En effet, dans notre exemple, la discussion qui sera établie entre les jeunes et les professionnels permettra de les faire adhérer au placement. Plus qu'un achat de la paix sociale, cette responsabilisation facilitera l'accompagnement des mineurs vers un projet de sortie de l'établissement et plus largement de sortie de la spirale judiciaire.

Ici, l'approche d'Erving Goffman semble pertinente à mobiliser. Il a éclairé, dans son œuvre sur les asiles psychiatriques, l'écart existant entre les normes prescrites et leur application. Il souligne ainsi la part imprévisible des institutions, étant notamment la conséquence d'une « adaptation secondaire » par les acteurs (Goffman, 1968). On perçoit ici que les acteurs recomposent, en dépit d'un contexte contraint, des marges de manœuvre et des capacités d'actions. Les intervenants provoquent le changement en ajustant les règles pour qu'elles répondent davantage aux besoins des pratiques professionnelles. Il est intéressant de noter que ces adaptations prennent le plus souvent place dans des espaces intermédiaires.

En effet, concernant les mineurs s'étant bagarrés, aucune délibération officielle n'a eu lieu suite aux événements de la journée. La réunion d'équipe hebdomadaire n'a lieu que quelques jours après. Si ces événements sont susceptibles d'être rediscutés dans des espaces formels de délibération, il faut proposer une réponse collective rapidement au risque de banaliser les violences de la journée. Ainsi, des échanges informels entre les professionnels

\footnotetext{
${ }^{3}$ Recherche intitulée : «Les Centres éducatifs fermés : La part cachée du travail éducatif en milieu contraint ». Elle est portée par l'Institut régional et européen des métiers de l'intervention sociale de Rhône-Alpes (Ireis) et financée par la mission de recherche Droit et Justice (Ministère de la Justice et CNRS). Coordonnée par Catherine Lenzi et Philip Milburn, cette recherche a mobilisé une équipe de 9 personnes (Catherine Lenzi, Philip Milburn, Yves Darnaud, Daniel Lepecq, Frédéric Mazereau, Sandrine Sanchez, Nordine Touil, Luc Trouiller et Léo Farcy-Callon).
} 
présents ont permis de prendre des décisions et de répondre aux violences le soir même. De nombreuses discussions seront établies avec les mineurs et le conflit ne prendra pas plus de proportions. Les professionnels seront plus à même de travailler sur leur situation. De manière pragmatique, le fait de sortir du cadre de l'intervention peut apaiser et prévenir les violences, qui comme nous l'avons vu, sont omniprésentes dans ces lieux.

On perçoit ici la force de l'informel et sa capacité à prendre part aux organisations et à réguler les institutions (Reynaud, 1989). Il s'avère qu'au-delà des protocoles, au-delà du travail prescrit, se développe, un ensemble de pratiques mises en place par les intervenants qui ne sont pas visibles dans les textes et différents documents administratifs. Comme nous le montre Anselm Strauss, les acteurs adaptent leur intervention, sur le modèle de l'ordre négocié, pour garantir une certaine stabilité. Ils provoquent le changement en ajustant les règles pour qu'elles répondent davantage aux besoins des pratiques professionnelles (Strauss, 1991).

\section{Conclusion}

Nous avons pu voir dans cet article que les brutalités physiques, le langage cru et l'agressivité sont aussi présents que les violences symboliques dans cette institution. La présence de ces phénomènes est telle qu'ils semblent être institutionnalisés et intériorisés par les acteurs, au point qu'ils en deviennent des banalités.

L'élément central de notre travail est de mettre en lumière la capacité des acteurs à mobiliser des savoirs faire pour gérer au mieux ces violences et tenter de minimiser leurs conséquences. Les jeunes, exacerbés par leur situation pénale, le peu de perspective et le manque de ressources, s'adaptent en trouvant des failles leur permettant de s'évader, en tentant de contourner les règles. Les professionnels, subissant fréquemment des violences verbales et physiques et étant soumis à la logique contraignante, développent des stratégies visant à la contenir et, outre mesure, à gérer au mieux la persistance de ces phénomènes. 


\section{Bibliographie}

Abbott, Andrew, The system of professions: An essay on the division of expert labor, Chicago, University Of Chicago Press, 1988.

Bailleau, Francis ; Gourmelon, Nathalie et Milburn, Philip, « Les établissements privatifs de liberté pour mineurs : entre logiques institutionnelles et professionnelles. Une comparaison entre EPM, QM et CEF », in Rapport de la Mission de Recherche Droit et Justice, janvier 2012.

Barreyre, Jean-Yves ; Fiacre, Patricia ; Joseph, Vincent et Makdessi, Yara, «Une souffrance maltraitée, parcours et situations de vie de jeunes dits incasables », ONED, Cedias, septembre 2008.

Bourdieu, Pierre et Passeron, Jean-Claude, La Reproduction. Éléments pour une théorie du système d'enseignement, Paris, éditions de Minuit, 1970.

Bourquin, Jacques, «Une histoire qui se répète : les centres éducatifs fermés pour mineurs délinquants », in Adolescence, n ${ }^{\circ}$ 4, Enfermement, avril 2005, pp. 877-897.

Caisne (1e), Leonore, Avoir 16 ans à Fleury, Paris, Seuil, 2008.

Champy, Florent, La sociologie des professions, Paris, PUF, 2009.

Chantraine, Gilles (dir.) ; Touraut, Caroline et Fontaine, Séverine « Trajectoires d'enfermement. Récit de vie au quartier mineurs », Rapport de recherche, DPJJ, Études et données pénales, $\mathrm{n}^{\circ}$ 106, Guyancourt, CESDIP, 2008.

Crettiez, Xavier, Les formes de la violence, Paris, La Découverte, 2008.

Dubet, François, Sociologie de l'expérience, Paris, Seuil, 1994.

Frauenfelder, Arnaud ; Nada, Éva et Bugnon, Géraldine, « Ce qu'enfermer des mineurs veut dire. Controverses professionnelles, conceptions éducatives et justification contemporaines de l'enfermement », Rapport de recherche, Genève, HETS, 2013.

Goffman, Erving, Asiles. Études sur la condition sociale des malades mentaux, Paris, Minuit, 1968.

Goffman, Erving, La mise en scène de la vie quotidienne, 1. La présentation de soi, Paris, Minuit, 1973.

Jaspart, Alice, Aux rythmes de l'enfermement. Enquête ethnographique en institution pour jeunes délinquants, Bruxelles, Bruylant, 2015.

Lenzi, Catherine et Milburn, Philip, «Les Centres éducatifs fermés : la part cachée du travail éducatif en milieu contraint », Rapport de recherche, 2015. 
Lenzi, Catherine et Milburn Philip, «Les centres éducatifs fermés : de la clôture institutionnelle à l'espace éducatif », in Espaces et sociétés, $\mathrm{n}^{\circ} 162$, Espaces d'enfermement, mars 2015, pp.95-110.

Martuccelli, Danilo, Forgé par l'épreuve, l'individu dans la France contemporaine, Paris, Armand Colin, 2006.

Martuccelli, Danilo, Sociologies De La Modernité, Paris, Gallimard, 1999.

Milburn, Philip, Quelle justice pour les mineurs? Entre enfance menacée et adolescence menaçante, Toulouse, Érès, 2009.

Mucchielli, Laurent, Violences et insécurité. Fantasmes et réalités dans le débat français, Paris, La découverte, 2001.

Mucchielli, Laurent, «Les centres éducatifs fermés : rupture ou continuité dans le traitement des mineurs délinquants? 》, Revue d'histoire de l'enfance "irrégulière », $\mathrm{n}^{\circ}$ 7, Enfermements et éducations, 2005, pp.113-146.

Nieburg, Harold L., Political Violence : The Behavioral Process, New York, St Martin's Press, 1969.

Potin, Émilie, Enfants placés, déplacés, replacés : parcours en protection de l'enfance, Toulouse, Érès, 2014.

Reynaud, Jean-Daniel, Les règles du jeu. L'action collective et la régulation sociale, Paris, Armand Colin, 1989.

Rostaing, Corinne, «L'ordre négocié en prison : ouvrir la boîte noire du processus disciplinaire. », in Droit et société, $\mathrm{n}^{\circ}$ 87, 2014, pp. 303-328.

Rostaing, Corinne, «La compréhension sociologique de l'expérience carcérale », in Revue européenne des sciences sociales, n XLIV, 2006, pp. 29-43.

Roux, Sébastien, «La discipline des sentiments. Responsabilisation et culpabilisation dans la justice des mineurs », in Revue française de sociologie, $\mathrm{n}^{\circ} 53,2012$, pp. 719-742.

Strauss, Anselm, La trame de la négociation. Sociologie quantitative et interactionniste, Paris, L'Harmattan, 1991.

Van Campenhoudt, Luc ; Chaumont, Jean-Michelet et Franssen, Abraham, La méthode d'analyse en groupe, applications aux phénomènes sociaux, Paris, Dunod, 2005.

Vidal-Naquet, Pierre, «Le travail de Care : tact, ruse et fiction » in Recherches en soin infirmiers, $\mathrm{n}^{\circ} 114,2013$, pp. 7-13. 